\title{
Coulisses
}

Revue de théâtre

14 | Printemps 1996

Varia

\section{L'homme derrière l'auteur de théâtre, le Tchekhov de Brook}

Ouriel Zohar

\section{OpenEdition}

1 Journals

Édition électronique

URL : http://journals.openedition.org/coulisses/4513

DOI : $10.4000 /$ coulisses. 4513

ISSN : 2546-9460

Éditeur

Presses universitaires de Franche-Comté

\section{Édition imprimée}

Date de publication : 1 mai 1996

Pagination : 22-28

ISSN : 1150-594X

\section{Référence électronique}

Ouriel Zohar, «L'homme derrière l'auteur de théâtre, le Tchekhov de Brook », Coulisses [En ligne], 14

Printemps 1996, mis en ligne le 20 mars 2019, consulté le 28 octobre 2019. URL : http://

journals.openedition.org/coulisses/4513; DOI : 10.4000/coulisses.4513

Ce document a été généré automatiquement le 28 octobre 2019.

Coulisses 


\title{
L'homme derrière l'auteur de théâtre, le Tchekhov de Brook ${ }^{1}$
}

\author{
Ouriel Zohar
}

1 De nos jours, lorsque l'on songe à Anton Tchekhov, nombreux sont ceux qui le considèrent comme un nouveau type d'homme dont notre siècle aurait tellement besoin. Peter Brook adhère certainement à cette opinion et comme beaucoup, il est certainement désolé qu'un tel type d'homme n'existe pas encore.

2 En 1981, Peter Brook montait La Cerisaie de Tchekhov au Théâtre des Bouffes du Nord à Paris. Des acteurs connus, comme Michel Piccoli, la femme de Brook, Natasha Parry et d'autres, participèrent à la production. Maurice Bénichou contribua à la mise en scène et joua le rôle de Yacha. Il fait toujours partie du groupe de recherche de Brook alors que dans le rôle de Lopakhine, Niels Arestrup était là pour la première fois. Brook sentait qu'il devait trouver pour cette œuvre une distribution composée d'acteurs issus de générations et de formations théâtrales différentes, pour parvenir à cette richesse que crée l'alliage de leurs diverses perceptions et de celles qui peuvent jaillir des méthodes de Constantin Stanislavski, avec le travail de l'acteur sur lui-même ${ }^{2}$. Lors d'une interview donnée à la télévision française sur T.F.1 le 3 avril 1981, Brook dit néanmoins :

J'ai recherché à travers les particularités de chacun, ce qu'ils avaient cependant en commun. Ce que nous possédions ensemble, ce n'était pas seulement un amour réel pour le travail théâtral, mais un même désir de produire quelque chose qui soit humain et vrai. Chacun des acteurs vint aux Bouffes du Nord avec le dessein de coopérer avec les autres de manière à ce que la pérennité de la vérité humaine soit exprimée et non pas dans le but de la reconnaissance d'une gloire personnelle ou égocentrique. Ceci peut paraître simpliste, mais c'est tout simplement ce qui arriva.

3 À l'époque, nous avons recueilli quelques commentaires auprès des acteurs, au moment où le théâtre, sous la direction de Micheline Rozan, était sur le point de produire la pièce de Tchekhov. Certains acteurs auraient préféré travailler avec Brook dans le cadre du Centre International de Créations Théâtrales, plutôt que de voir monter sur leur scène des acteurs parisiens, même célèbres, n'ayant jamais participé auparavant au théâtre Laboratoire. Leurs difficultés à accepter les conditions imposées par Brook 
laisse néanmoins percevoir leur haute opinion à l'égard de son travail et les difficultés qu'ils éprouvent tous à renoncer à leur participation à chacune des pièces interprétées sous sa direction.

\section{Le principe de fidélité}

4 Brook admet avoir lu cinq ou six versions françaises de La Cerisaie, encore bien davantage de traductions en anglais et évidemment le texte original en russe. Comment est-ce possible ? Étudiant à Oxford, il apprit le russe grâce à ses parents. Durant son enfance, ses parents d'origine russe, lui parlèrent beaucoup de leur pays et sa mémoire resta gravée dans son cœur. Quant à sa femme Natasha Parry, elle ne coupa jamais ses liens avec la Russie. La mère de Natasha parlait le russe, tout au long du travail avec elle, Peler Brook dirigea la lecture de la pièce. Avec Jean-Claude Carrière il créa une nouvelle adaptation de l'œuvre. Selon lui, la nécessité et le besoin de réadapter les pièces de Tchekhov existeront toujours, pour découvrir des points de vue différents de l'époque où elles furent représentées et pour recueillir les influences de la société du moment, l'influencer aussi.

L'orientation scientifique de Tchekhov influençait aussi ses conceptions idéologiques et sa philosophie de vie. Il était médecin et se présentait toujours comme tel. Nous devons néanmoins signaler qu'il ne fut jamais un scientifique dogmatique et théorique. De plus, il était offusqué par l'arrogance qu'il constatait trop souvent dans les salles de conférences et les laboratoires qu'il fréquentait. Sans doute en réaction à cet état de fait, nous le supposons, il s'est toujours montré très actif et dévoué à ses patients. Son but essentiel dans la vie était le soulagement immédiat qu'il pouvait apporter à ses malades.

6 Peter Brook décida de monter la pièce de Tchekhov parce qu'il croyait en elle, précisément à cause des qualités de « fidélité » de cette famille. Cela contraste avec la pièce La Conférence des Oiseaux, montée à Paris en 1979, où il mettra plutôt l'accent sur l'imprévu. Durant les dix années consacrées à la préparation de cette pièce, il laissa libre cours à l'improvisation grâce à des exercices nouveaux sur un texte écrit par le poète Farid Audin Attar. Avec La Cerisaie, Brook préféra s'intéresser à un processus ne livrant pas d'obscurité, et où chaque mot sera clarifié et employé avec exactitude.

7 La précision est l'une des plus importantes qualités de l'auteur Tchekhov. Brook a plus d'une fois comparé le style poétique de Tchekhov avec les capacités esthétiques du cinéma qui permettent aux scènes de s'entrelacer naturellement et harmonieusement avec une grande précision. En tant qu'écrivain, Tchekhov recherchait les qualités faites de naturel et attendait que le jeu et la direction scéniques soient aussi transparents que la vie. Pour obtenir le maximum de crédibilité et réussir à créer une atmosphère juste, il était nécessaire de doter le texte français de qualités littéraires inexistantes dans la version russe où le niveau de langage est plus simple. En effet, dans la langue russe, les qualités littéraires s'expriment même avec une formulation rudimentaire. Pour obtenir le même effet en français, il fallait utiliser un style plus sophistiqué alors que dans la langue russe même une écriture simple laisse transparaître de la poésie.

8 L'écriture de Tchekhov est très concentrée. Ses personnages expriment leur monde intérieur avec une étonnante économie de mots. Nous remarquons la même tendance à la sobriété chez des écrivains contemporains comme Harold Pinter et Samuel Beckett. 
Pour eux aussi la structure, le rythme et la pureté poétique théâtrale sont primordiaux. Ils savent utiliser le mot juste, à sa juste place. Certains acteurs dans le théâtre de Brook, comprennent les effets de chaque mot, au sens le plus pratique.

Par exemple, ils sont capables d'utiliser le mot «oui » de telle manière que "oui » tourne à une parfaite expression qui ne peut être remplacée par aucun autre terme.

L'attachement de Tchekhov à la précision de l'écriture d'un mot, fit surgir de multiples questions dans l'univers des critiques théâtraux. Est-il constamment et durablement précis? Cette question fut principalement traitée dans une lettre qu'il écrivit en 1903, contenant d'ailleurs de piquantes critiques à l'égard de la langue française. Le critique théâtral français qui lit aujourd'hui la lettre de Tchekhov est confondu par la vérité de son contenu. Mais du point de vue théâtral, certains diront que si Tchekhov écrivait cette lettre aujourd'hui, dans le Théâtre des Bouffes du Nord sous l'influence de Peter Brook, il écrirait cette correspondance de telle sorte que la signification de chaque mot puisse changer à tout moment, parce que la conception même du théâtre de Brook est faite de changement permanent en continuelle mobilité comme la vie elle-même. C'est le sens du théâtre en 1981, avec la production de La Cerisaie, comme une réponse à ceux qui affirment qu'il y a certaines obscurités dans les mots de Brook, ou dans les autres pièces de Tchekhov.

11 Les Français que Tchekhov rencontra durant ses voyages au début du siècle sont différents de ceux d'aujourd'hui. Ainsi les pièces de Brook ne sont pas les mêmes que celles que produisait Stanislavski à Moscou.

\section{La Cerisaie. Mise en scène Théâtre d'Art de Moscou. 1904}

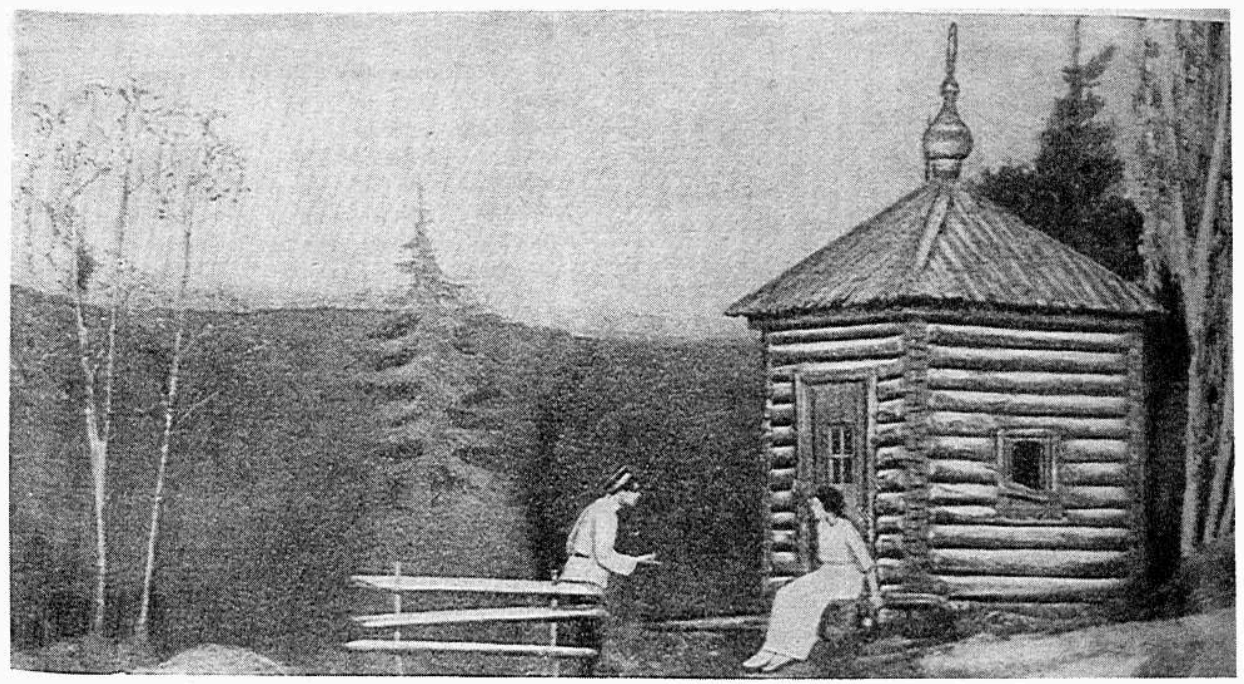

Il ne faut pas conclure pour autant que Brook abandonna sa fidélité à l'égard de l'un des plus grands maîtres du théâtre contemporain. Bien au contraire, Brook est fidèle comme toujours aux conceptions théâtrales de Stanislavski et précisément en ce qui concerne le travail de l'acteur sur sa vie intérieure. C'est pour cette raison qu'il est devenu l'un des plus grands disciples de celui-ci. Brook est en quête d'une nouvelle rencontre : la rencontre de ceux qui jouent la pièce ici et maintenant avec le public d'aujourd'hui. Pour nous, il existe encore une réelle attente, celle de redécouvrir Tchekhov de nos jours mais avec un langage, un style et une forme correspondant à 
notre modernité. C'est vraisemblablement pourquoi Brook a traduit à nouveau et réadapté cette pièce.

À la fin du livre présentant le texte de la pièce, nous distinguons quelques photographies impressionnantes du spectacle de Stanislavski au théâtre artistique de Moscou. La première a lieu le 17 janvier 1904. (L'adaptation de la pièce de J.-C. Carrière, publiée par le Centre International de Création Théâtrale, sous les auspices de Micheline Rozan, eut lieu le 5 mars 1981). Stanislavski lui-même joua le rôle de Léonid Andrevich Gaiev et apparaît sur l'une de ces photos (rôle joué par Michel Piccoli chez Brook). Selon Brook, la pièce remporta un grand succès à ce moment-là, parce que Tchekhov était déjà très connu. Néanmoins il y eut une sérieuse discussion entre Tchekhov et Stanislavski. Tchekhov, affirmant que la pièce était comique, était mécontent de la mise en scène de Stanislavski, trop sérieuse à son goût. Mais selon Brook, Stanislavski fit un travail de mise en scène extraordinaire et il suffit pour s'en convaincre de regarder les photographies de l'époque.

D'après Stanislavski, il s'agit d'une tragédie. C'est pourquoi il en fait la mise en scène à un rythme beaucoup plus lent avec une interprétation plus triste que ne l'avait désiré l'auteur. Néanmoins le style naturaliste du théâtre de Moscou amena la pièce au succès que nous connaissons. Les acteurs jouèrent merveilleusement bien et Brook se référant toujours aux illustrations, commente les photographies et remarque que nous pouvons voir la qualité du jeu de Stanislavski dans le rôle de Gaiev. «Il suffit de regarder son visage pour reconnaître les qualités humaines ayant contribué à façonner son caractère. Donc malgré les différences d'opinion entre Tchekhov et Stanislavski, les deux partagent cette qualité humaine... » (tiré de l'interview déjà mentionnée).

Puisque Brook avait décidé d'adopter le principe de fidélité, il devait aussi vérifier et rechercher si la version française était fidèle au texte original russe. Là résidait le danger de voir l'adaptation perdre en profondeur et devenir artificielle en utilisant des synonymes ne traduisant pas exactement les mots désirés. Si la similarité des mots est peut-être possible dans l'écriture littéraire, le langage russe ne peut être transformé. Ainsi l'adaptation de Brook et de J.-C. Carrière est une tentative de donner aux acteurs des mots et des outils précis, des phrases et des structures exactes, sur lesquels ils puissent fonder l'évolution mentale de leur pensée. Brook signale que tous les acteurs respectèrent fidèlement cette structure au point et à la virgule près.

\section{Tchekhov : la perfection des contraires}

Tchekhov est perçu par beaucoup comme un écrivain mélancolique. C'est un romantique et ses pièces sont pleines d'états d'âme nuancés et variés. Peter Brook considère son œuvre comme une totalité dans laquelle il est possible de tout trouver. Mélancolie et états d'âme, mais pas uniquement cela.

Il faut le prendre comme s'il y avait tout dans Tchekhov... C'est la qualité de la pièce et par conséquent on peut dire que l'œuvre est semblable à la vie elle-même. Nous pouvons la ressentir et même il existe l'obligation de voir la pièce d'un millier de points de vue différents et cela simultanément. Il est clair que tout existe en elle et aussi la mélancolie slave. Mais à partir du moment où cet aspect est le seul qui soit amplifié, la pièce perd son côté naturel. Exactement comme dans la vie où l'on devient non naturel lorsque l'on n'y trouve que de la mélancolie et des amusements. Dans les pièces de Tchekhov nous passons constamment du rire aux larmes, d'une chose indéfinie et impossible à exprimer à une autre qui est très 
précise et spécifique. Nous sommes alors au cœur de la vie sociale, la vie sous sa forme politique la plus concrète contenant en même temps une symbolique et une pure poésie. Ces deux directions de la littérature et de la politique, sont capables de nous faire comprendre pourquoi les pièces de Tchekhov sont si fortes, tellement amples qu'elles nous apportent le sentiment de la vie et de la vérité en même temps. $^{3}$

Le véritable désir de Tchekhov était celui d'un écologiste voulant vivre dans un environnement plus net, plus propre, plus brillant, pour un mieux-être, et que tous soient heureux et vivent dans la félicité. Alors qu'à la même époque, les autres intellectuels fréquentant les salons de la Russie profonde vivaient une existence d'extrême scepticisme, Tchekhov refusait pour sa part de servir une seule et même idéologie. D'un côté, il ressentait la nécessité d'être pragmatique, mais d'autre part il savait utiliser le scepticisme :

Entre Dieu existe et Dieu n'existe pas il y a un espace immense que l'homme sage et sincère traverse avec une grande difficulté. Le Russe ne connaît qu'un de ces extrêmes car ce qu'il y a entre les deux ne l'intéresse pas. C'est pourquoi d'ordinaire il ne sait rien ou presque rien. ${ }^{4}$

Mais il ne faudrait pas se méprendre sur la personnalité du docteur Tchekhov. C'est un médecin praticien ayant réussi à associer la sensibilité d'un artiste qui a foi dans l'avenir de l'homme de science, car il est convaincu que l'instruction et l'esprit scientifique sortiront l'homme de son ignorance, de sa paresse, de sa brutalité et de sa souffrance. Ces contradictions sont très bien entremêlées grâce à son talent d'écrivain et l'on ne peut douter de sa sensibilité littéraire. Il est vrai qu'il n'est pas le seul médecin à devenir écrivain, mais lequel a écrit pour le théâtre comme lui ? D'autres auteurs peuvent observer comme lui; ils ont eu des expériences sociales riches et similaires, mais qui parmi eux possède une telle tendresse et tant de compassion à l'égard de la souffrance humaine, une humanité si profonde? Quels sont ceux qui écrivent avec une telle exactitude, sachant éliminer tout ce qui est superflu dans le texte et créer une atmosphère aussi spéciale pour décrire un personnage, une situation et représenter avec si peu de mots un monde entier si complexe.

Selon Peter Brook, c'est là que réside la grande distinction entre le théâtre et la vie :

Dans la vie nous sommes tous occupés à ne considérer qu'une partie de notre point de vue, d'une manière tellement puissante qu'il est très difficile de nous en faire sortir. Dans le théâtre nous sommes libres d'être en même temps partie prenante et objectif. Si l'acteur joue bien et si la pièce a une puissance, nous recevons son point de vue de manière absolue. Mais au moment où il s'arrête de parler, nous sommes libres de pénétrer de manière intégrale dans le corps et l'âme de l'autre et d'accepter l'autre point de vue. C'est pourquoi dans la pièce d'un auteur exceptionnel comme Tchekhov, nous vivons de manière très intensive le point de vue contradictoire. De cette façon, le public n'est jamais un juge mais un participant actif. Quand le caractère est joué de manière juste, nous ne sommes ni avec lui ni contre lui, nous sommes comme l'auteur, nous le comprenons. Et nous saisissons en même temps, avec le même sérieux et la même force aussi, celui auquel il s'oppose, l'antagoniste. ${ }^{5}$

20 Ici la confrontation des forces ne se situe pas uniquement entre un personnage et un autre. C'est une opposition semblable à celle qui existe dans la personnalité de Tchekhov, au moment même de l'écriture et de la création. L'oscillation du public, sa participation et son identification à la tristesse d'un personnage, passe par un transfert avec un personnage antagoniste. C'est déjà le troisième niveau de la confrontation: celui de l'écrivain avec lui-même, un personnage avec un autre, l'observateur du public 
avec lui-même. Mais il existe encore d'autres niveaux. C'est la pensée de l'identification de chaque acteur avec l'écrivain, ou bien avec le personnage qu'il joue ou encore avec le personnage antagoniste à celui qu'il joue, ou bien avec le metteur en scène... Sur cette question il y a déjà une réponse au moins partielle dans la personnalité et l'activité de Tchekhov. Lorsque l'on observe le caractère de Tchekhov, on remarque d'une part une attitude et des méthodes scientifiques et de l'autre, des antennes réceptives sensibles et délicates, néanmoins pratiques et vivantes, comme celles qui existent dans l'ensemble de la littérature russe.

21 L'emploi du temps de Tchekhov nous donnera peut-être une idée de cette perfection sociale, politique, médicale et artistique. Le matin, il ouvrait gratuitement sa clinique et donnait des traitements aux fermiers qui venaient le consulter. L'après-midi, il était penché sur les plans d'élargissement et d'amélioration des jardins publics, la transformation de l'école et le développement de la bibliothèque publique et le soir, il écrivait des chefs-d'œuvre d'une valeur artistique à l'égal des plus grands classiques. Tout cela sans dogmatisme ni théorie, sans cette amertume refoulée que l'on rencontre souvent chez les idéologues qui ne réussissent pas à atteindre leur idéal. Il faisait tout avec un calme d'esprit, une délicatesse, un humour plaisant, une grande miséricorde et compassion. Ce qui nous ramène tout naturellement à notre introduction : Tchekhov est probablement un nouveau type d'homme.

\section{L'auteur d'un mouvement de vie et de mort}

22 L'habileté de l'acteur, ou même du public, de passer à l'identification de l'un ou l'autre des caractères, touche aussi la capacité temporaire de l'auteur, et peut-être aussi de l'homme en général, à vivre dans une sorte d'harmonie des contraires. La Cerisaie est une pièce exceptionnelle, parce qu'il y a là des gens qui essaient de trouver leurs qualités propres dans la rencontre des contraires et des plus grandes contradictions de l'époque russe d'avant la révolution. Toutefois, ces antagonismes, dans le sens d'un conflit direct entre les personnages sur la scène, peuvent absolument convenir à notre époque. 


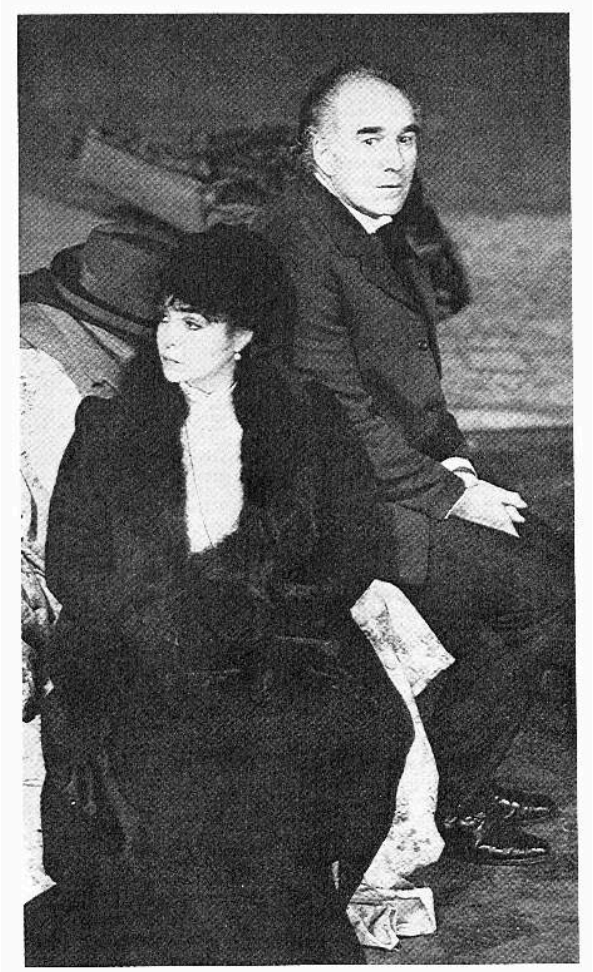

Ranevskaïa et Gaïev

Photo F. Darras

Brook voit Tchekhov comme un parfait monteur de film sauf que Tchekhov, au lieu de couper le film pour passer d'une image à l'autre, crée une sorte de transition émotionnelle. Toujours, un moment avant que tout soit exprimé, à l'instant où le spectateur peut s'identifier avec l'un des personnages plus qu'avec un autre, il change soudainement la situation de manière à créer d'autres inattendus et un brusque sentiment d'instabilité.

En tant qu'auteur de théâtre, Tchekhov approfondit le sens du changement en nous présentant des individus dans une société en perpétuel état de mutation. C'est pourquoi nous le voyons comme l'auteur du mouvement de la vie, sérieux et souriant, amer et drôle en même temps. Si l'on accepte cette supposition, alors il est plus facile d'intégrer l'idée de Brook lorsqu'il déclare que le travail de Tchekhov contient tout et que c'est aussi la qualité de la pièce. Tchekhov ne peut ignorer les contradictions dans lesquelles il vivait quotidiennement et il les a trouvées aussi chez les autres. Il observe les détails les plus infimes de la comédie humaine. Parce que lui-même regardait la vie comme une comédie, il attendait de Stanislavski qu'il mette en scène La Cerisaie comme une comédie. L'homme Tchekhov était très actif et voyait à la fois le côté triste et joyeux de la vie. Il refusa le style dramatique et lent imposé à la pièce par Stanislavski, qui selon lui, apportait une fausse note à la perfection de La Cerisaie, dans laquelle il avait voulu aborder les deux extrêmes de la contradiction et non pas l'un des aspects uniquement.

En qualité de médecin, Tchekhov savait très bien observer les attitudes humaines et le sens du comportement des hommes ne lui échappait pas. Cette qualité lui permettait de discerner l'essentiel pour trouver le bon diagnostic. Il faut bien distinguer chez lui le 
sentimentalisme, qui n'existe pas du tout dans son travail, et sa délicate et sensible tendance à une sympathie attentive. Peut-on imaginer un médecin versant des larmes sur les souffrances de ses patients? Ce serait plutôt maladroit et comique. Par contre, nous pouvons l'imaginer comme un amoureux sachant discerner mieux que les autres les lacunes et les souffrances de sa bien-aimée, sans crainte, sans se fâcher et même avec un sourire.

La fidèle minutie de Tchekhov, son attention au moindre détail des caractères, son style et son expression sont différents de Shakespeare. Chez Shakespeare, les phrases sont comme des télégrammes et les acteurs doivent les regrouper et trouver le rythme des mots et des accents. Avec Tchekhov, c'est le contraire. Les points, les virgules et les points d'exclamation ont une importance prédominante, comme chez Beckett, le temps dicte le rythme de la pièce et ses expressions. Chez Tchekhov la ponctuation présente un groupe de messages exacts, décrivant les relations et les sentiments des personnages, les moments où les idées s'entrelacent ou fonctionnent toutes seules. La ponctuation chez Tchekhov permet de saisir le sous-texte, ce qui est caché derrière les mots et les phrases.

En tant que médecin, il était très souvent confronté avec la mort, mais nous ne trouvons chez lui ni sa négation, ni le mépris dans le style des carnavals grotesques de certains maîtres flamands. Dans ses pièces, la présence de la mort est permanente et représente une sorte de mutation et de changement menaçant à chaque instant la situation. Cette conscience de la mort contient aussi son contraire absolu : un immense besoin de renforcer le désir de vie. Ici, les personnages ont conscience de renforcer la notion « d'ici et maintenant » comme un événement, une part essentielle de la vie qu'il faut sauver. Cette idée de la confrontation entre la vie et la mort trouve chez Tchekhov son équilibre parfait entre une création nouvelle et sa disparition. Ainsi, chaque contradiction est équilibrée et donne à sa création cette "aura » de perfection qui existait dans les grandes tragédies classiques.

Tchekhov est mort jeune après avoir voyagé dans le monde. Il a écrit, aimé et participé à la planification et l'amélioration de sa société. Sa conscience de la mort et des moments précieux de la vie lui donnèrent son sens aigu de la relativité, c'est-à-dire une distance suffisante pour ne pas perdre le point de vue caucasien du drame et de la tragédie.

29 «Si l'on ne veut pas trahir son travail, dit Brook à la fin de la pièce ${ }^{6}$, il faut faire coïncider ces deux éléments de la vie et de la mort. Si l'on veut être fidèle à celui qui a inventé le théâtre moderne, il faut nécessairement inventer aussi ». Cette immense admiration de Brook provient de la connaissance approfondie qu'il a de Tchekhov. Il déclare aussi que pour en effectuer les mises en scène, il n'est pas suffisant d'utiliser ce qui a été dit ou déjà mis en scène, avant et après Tchekhov.

L'unicité de Tchekhov sera sans doute mieux perçue par une société ayant un sens de l'observation exercé. Ainsi, Brook décida de faire plusieurs répétitions avec sa troupe devant une communauté de sourds-muets, personnes devant se confronter à la vie malgré leurs sens éteints et morts et qui possèdent néanmoins une capacité à voir des phénomènes échappant à la majorité des hommes. Ces rencontres ont beaucoup soutenu la troupe afin d'améliorer son jeu et surtout permettre aux acteurs de mieux s'observer eux- mêmes :

Je n'ai jamais vu chez aucun autre public cette sorte d'humour calme et généreux.

C'est quelque chose d'absolument unique car ils passent leur vie à observer. Et 
comme rien ne les distrait, rien ne leur échappe, et surtout pas l'absurdité des gestes inutiles. ${ }^{7}$

\section{Mise en scène dans un mouvement renouvelé}

31 Chaque personnage de Tchekhov possède sa propre existence et son autonomie, aucun ne ressemble à l'autre. L $\boldsymbol{a}$ Cerisaie représente le microcosme des tendances de l'époque. Certains personnages annoncent des changements sociaux, d'autres expriment le regret d'événements en voie de disparition. Brook ne les observe pas du point de vue extérieur et intellectuel uniquement. Il les regarde de l'intérieur, depuis leur monde intime dans lequel il découvre des aspirations permanentes et des désirs en éveil. Les personnages ne font pas d'efforts pour exposer leurs erreurs, bien au contraire. Chaque personnage dans la pièce essaie à sa manière de présenter ce qu'il a de plus sublime dans le domaine émotionnel et social. Ils n'ont aucune crainte d'exister simultanément dans deux mondes positifs et négatifs et ainsi ils vivent pleinement la diversité des changements nuancés de leurs sentiments, comme au cinéma lorsque l'on passe d'une image et d'une situation à l'autre. Leur drame vient du fait que le monde extérieur ne leur permet pas ce passage tranquille et qu'il met toute sa lourdeur pour empêcher cette liberté. Malgré cette tension, les personnages sont rarement destructifs. De la même façon, la mise en scène de Brook permet à la fois de reconnaître le conflit intérieur des personnages et de présenter leur façon, parfois même ridicule, d'exposer leurs qualités humaines. La complexité des comportements très énigmatiques des acteurs et des personnages de Tchekhov ne s'exprime pas par des mots dits ou écrits. La complication se développe et résulte d'une structure faite de mosaïques bien définies et fabriquées, tout d'abord par l'auteur, ensuite grâce à l'orientation du metteur en scène et enfin par l'adaptation. Cette structure est constituée d'une multitude de détails laissant apparaître la psychologie des personnages jusqu'à ce que leur rayonnement passe vers le public.

Pour faire la mise en scène de cette pièce, chaque détail fut travaillé le plus minutieusement possible. De cette manière, l'objet le plus insignifiant recevait la forme désirée par le metteur en scène, ceci tout particulièrement grâce au travail de laboratoire que la majorité des acteurs eut la chance de suivre avec Brook à Paris durant les dix ans qui précédèrent La Cerisaie. Brook définit cette sorte de travail de laboratoire comme la recherche théâtrale, mais nous savons tous combien il est difficile d'entrer dans le laboratoire de Brook et le connaître de l'intérieur. C'est une véritable quête. Lorsque l'on pose la question à Brook sur la mise en scène de La Cerisaie, il répond, sans doute par humilité et désir de perfection, qu'il est peut-être impossible de trouver un metteur en scène capable de faire un jour une mise en scène absolue de Tchekhov. De manière analogique, il prend alors l'image du bébé et demande si l'on peut imaginer un bébé absolu et parfait. Le fotus se développe durant neuf mois pour parvenir à une forme définie. Après sa naissance, il changera encore. Il sera influencé par son entourage, son époque et se développera indépendamment.

Pour Brook, le texte dramatique écrit est une chose mystérieuse. C'est une abstraction qu'il faut habiller d'un contenu ici et maintenant. Avec Brook, les acteurs doivent constamment examiner leurs potentialités cachées et la connaissance qu'ils ont d'euxmêmes pour pénétrer un texte complexe et difficile à interpréter. Brook participe luimême avec ses acteurs à sa propre recherche. Ensemble, ils enrichissent leurs 
potentialités. Peu à peu, ils créent en commun quelque chose qu'il définit comme le " témoignage objectif ». Le contact avec Brook se fait toujours sur le mode idéaliste, car le théâtre est la rencontre d'un groupe, d'un lieu et d'un texte infini. Cette réflexion n'apparaît que lorsque les hommes de théâtre deviennent plus sensibles, grâce au développement de leurs potentialités et une meilleure connaissance d'eux-mêmes. Ils s'ouvrent aussi aux changements de la société, à la souffrance des autres, à leurs contemporains et à leur époque. C'est précisément dans cette identification qu'une sorte d'énergie collective permet de réaliser la rencontre avec le public: "la rencontre " au sens de Brook. Le témoignage objectif n'a de valeur que durant les répétitions, les exercices et les représentations. Durant cette période, l'acteur développe ses liens et ses mouvements permanents entre vie privée et intime et une capacité d'utiliser l'expérience de sa vie intérieure pour la représenter au public. C'est à ce moment-là qu'il est témoin du texte, du metteur en scène et du personnage qu'il joue. C'est là aussi le sens de la représentation pour Brook. Ensuite, ils devraient tous se réunir à nouveau et tout recommencer.

\section{NOTES}

1. Une version anglaise de ce texte est disponible. La demander à Coulisses.

2. Constantin Stanislavski, La formation de l'acteur, préface de Jean Vilar, Petite Bibliothèque Payot, Paris, 1979.

3. Peter Brook, À propos de Tchekhov, La Cerisaie, collection Créations Théâtrales, publiée sous la direction de Micheline Rozan, Paris, 1981, pp. 107-110.

4. J.-P. Priestley, Sur Tchekhov, introduction de la pièce La Cerisaie, pp. 9-10.

5. Interview avec Peter Brook, T.F.1, 3 avril 1981.

6. La Cerisaie, p. 110. Propos recueillis par la critique Colette Godard, pour la revue ComédieFrançaise de février 1981, Paris.

7. Ibid. p. 111

\section{AUTEUR}

\section{OURIEL ZOHAR}

Professeur à l'université de Tel-Aviv, contribue régulièrement à Coulisses 